\title{
A Disintegrin and Metalloproteinase With Thrombospondin Motifs 13
}

National Cancer Institute

\section{Source}

National Cancer Institute. A Disintegrin and Metalloproteinase With Thrombospondin

Motifs 13. NCI Thesaurus. Code C118327.

A disintegrin and metalloproteinase with thrombospondin motifs 13 (1427 aa, 154 kDa) is encoded by the human ADAMTS13 gene. This protein plays a role in the proteolysis of von Willebrand factor multimers. 